Pacific Journal of Mathematics

TOPOLOGICAL MEASURE CONSTRUCTION

June 1963 


\title{
A TOPOLOGICAL MEASURE CONSTRUCTION
}

\author{
W. W. Bledsoe and A. P. Morse
}

1. Introduction. The purpose of this paper is to give two results in topological measure theory that generalize two well known results for metric spaces.

The principal one of these, which is given in $\S 3$, concerns the construction of a measure from a nonnegative set function. Carathéodory [1] has done this in a natural way in defining Carathéodory linear measure in a finite dimensional Euclidean space. It is well known that this Carathéodory construction can be applied in metric spaces to produce measures for which the open sets are measurable. ${ }^{1}$ Our treatment produces a measure for which the open $F_{\sigma}$ sets, in a regular topological space, are measurable, and is identical with the Carathéodory measure in case the topology is metrizable. Since each open set in a metric space is an $F_{\sigma}$ this provides a generalization of the metric result.

Our other result, which is given in $\S 2$, concerns a necessary and sufficient condition for the measurability of open sets. A well known condition for this in metric spaces is that the measure be additive on any two sets which are a positive distance apart. When this condition is changed to require the additivity on two sets whose closures do not intersect, it becomes a necessary and sufficient condition for the measurability of the open $F_{\sigma}$ sets, for a normal topological space. We show that the condition of normality can be weakened to one of " $\phi$ Normal" (see definition 2.4.5 below). Since a metric space is normal and therefore $\phi$ Normal, and since each open set of a metric space is an $F_{\sigma}$, this provides a clear generalization of the metric result.

At first glance the weakened normality condition of 2.4 .5 appears to add little to topological measure theory. However, this is just the condition that results from our construction in $\S 3$ (even though the topology is not necessarily normal) and hence the results of $\S 2$ help us to obtain the results of $\S 3$.

Nowhere in this paper is an assumption of local compactness made.

\section{Conditions for measurability.}

\subsection{Definitions.}

Received February 13, 1963.

1 See, for example, Method II, page 105, of [3]. 
$.1 \sim B=$ the complement of $B$.

$.2 \sigma F=$ the union of $F=\mathrm{U} \beta \in F \beta=\mathrm{Ex}(x \in \beta$ for some $\beta \in F)$ $=$ the set of points $x$ for which $x \in \beta$ for some $\beta \in F$.

$.3 x \in \operatorname{domain} f$ if and only if $(x, y) \in f$ for some $y$.

$.4 \omega=$ the set of nonnegative integers.

\subsection{Definitions.}

$.1 \phi$ measures $\mathscr{S}$ if and only if $\phi$ is such a function of the subsets of $\mathscr{S}$ that:

$$
0 \leqq \phi(A) \quad \text { whenever } \quad A \subset \mathscr{S} \text {; }
$$

and

$$
\phi(A) \leqq \sum \beta \in F \phi(\beta)
$$

whenever $F$ is a countable family for which

$$
A \subset \sigma F \subset \mathscr{S} \text {. }
$$

$.2 A$ is $\phi$ measurable if and only if $A \in$ domain $\phi$ and

$$
\phi(T)=\phi(T A)+\phi(T \sim A)
$$

for each $T \in$ domain $\phi$.

.3 measurable $\phi=\mathrm{E} A(A$ is $\phi$ measurable $)$

.4 section $\phi T=$ the function $\psi$ on domain $\phi$ such that

$$
\psi(A)=\phi(T A)
$$

for each $A \in$ domain $\phi$.

$.5 \psi$ is a submeasure of $\phi$ if and only if $\psi=\operatorname{section} \phi T$ for some $T$ for which $\phi(T)<\infty$.

\subsection{Definitions.}

$.1 \mathfrak{T}$ is a topology if and only if $\mathfrak{T}$ is such a family of sets that

$$
\sigma F \in \mathfrak{T} \quad \text { whenever } \quad F \subset \mathfrak{I},
$$

and 


$$
\alpha \cap \beta \in \mathfrak{I} \quad \text { whenever } \alpha \in \mathfrak{I} \text { and } \beta \in \mathfrak{T} \text {. }
$$

Thus a topology $\mathfrak{I}$ is closed to finite intersections and unrestricted unions. For example, the family of all open sets of a metric space is a topology.

$.2 \mathfrak{I}$ topologizes $\mathscr{S}$ if and only if $\mathfrak{I}$ is a topology and $\mathscr{S}=\sigma \mathfrak{I}$.

$.3 \quad C$ is $\mathfrak{I}$ closed if and only if $C=\sigma \mathfrak{T} \sim A$ for some $A \in \mathfrak{T}$.

.4 Closure $\mathfrak{I} A=$ the intersection of all $\mathfrak{I}$ closed sets which contain $A$.

$.5 \mathfrak{I}$ is regular if and only if corresponding to each $A \in \mathfrak{T}$ and each $x \in A$ there is a $B \in \mathfrak{T}$ such that $x \in B$ and Closure $\mathfrak{I} B \subset A$.

.6 Fsigma $\mathfrak{I}=\mathrm{E} B(B \in \mathfrak{T}$ and $B=\sigma F$ for some countable family $F$ of $\mathfrak{T}$ closed sets).

.7 Gdelta $\mathfrak{I}=\mathrm{E} C(C$ is $\mathfrak{I}$ closed and $C$ is the intersection of a countable subfamily of $\mathfrak{I})$.

Thus Fsigma $\mathfrak{I}$ and Gdelta $\mathfrak{I}$ are the familiar open $F_{\sigma}$ 's and closed $G_{\delta}$ 's.

This paper deals with a fixed topology, $\mathfrak{I}$, which topologizes the space, $\mathscr{S}$. It is assumed that the hypothesis

\section{$\mathfrak{I}$ topologizes}

is added to every theorem. Also the "T⿱乛龰" will be dropped from such expressions as " $C$ is $\mathfrak{T}$ closed" whenever no confusion will result. Thus we write "Fsigma" for "Fsigma $\mathfrak{I}$," "Closure $A$ " for "Closure $\mathfrak{I} A$," etc.

In definitions 2.4 below the well known topological concepts of compactness and normality, are followed by generalizations involving both topology and measure.

\subsection{Definitions.}

$.1 A$ is compact if and only if $A$ is closed and for each $F \subset \mathfrak{I}$ for which $A \subset \sigma F$ there is a finite subfamily $H$ of $F$ for which $A \subset \sigma H$.

$.2 A$ is $\phi$ compact if and only if $A$ is closed and for each $F \subset \mathfrak{I}$ for which $A \subset \sigma F$, for each submeasure $\psi$ for $\phi$, for each $\varepsilon>0$, there is a finite subfamily $H$ of $F$ for which $\psi(A) \leqq \psi(A \sigma H)+\varepsilon$. 
$.3 A$ is normal if and only if $A$ is closed and for each $C$ and $B$ for which $C$ is closed, $C \subset A, C \subset B \in \mathfrak{T}$, there exists $D \in \mathfrak{I}$ for which $C \subset D$ and Closure $D \subset B$.

$.4 A$ is $\phi$ normal if and only if $A$ is closed and for each $C$ and $B$ for which $C$ is closed, $C \subset A, C \subset B \in \mathfrak{I}$, and for each submeasure $\psi$ for $\phi$, for each $\varepsilon>0$, there exists $D \in \mathfrak{I}$ and a closed set $C^{\prime}$ for which $C^{\prime} \subset C, C^{\prime} \subset D$, Closure $D \subset B$, and

$$
\psi(C) \leqq \psi\left(C^{\prime}\right)+\varepsilon
$$

We define the slightly less general notion of $\phi$ Normality by changing the condition (1) to condition (2) below.

$.5 A$ is $\phi$ Normal if and only if $A$ is closed and for each $C$ and $B$ for which $C$ is closed, $C \subset A, C \subset B \in \mathfrak{T}$, and for each submeasure $\psi$ of $\phi$, for each $\varepsilon>0$, there exists $D \in \mathfrak{I}$ and a closed set $C^{\prime}$ for which $C^{\prime} \subset C, C^{\prime} \subset D$, Closure $D \subset B$, and

$$
\psi\left(C \sim C^{\prime}\right) \leqq \varepsilon .
$$

$.6 \phi$ is $\mathfrak{I}$ additive if and only if $\phi(A \cup B)=\phi(A)+\phi(B)$ whenever Closure $A \cap$ Closure $B=0$.

$.7 \phi$ is a $\rho$ metric measure if and only if $\rho$ metrizes $\mathscr{S}, \phi$ measures $\mathscr{S}$, and $\phi(A \cup B)=\phi(A)+\phi(B)$ whenever $A$ and $B$ are subsets of $\mathscr{S}$ which are a positive $\rho$ distance apart.

2.5. TheoRem. If $\phi$ measures $\mathscr{S}$ then:

.1 if $A$ is compact then $A$ is $\phi$ compact;

.2 if $A$ is normal then $A$ is $\phi$ Normal;

.3 if $A$ is $\phi$ Normal then $A$ is $\phi$ normal.

2.6. THEOREM. If $\rho$ metrizes $\mathscr{S}, \mathfrak{I}$ is the family of $\rho$-open sets, and $\phi$ measures $\mathscr{S}$ then:

$.1 \phi$ is $\mathfrak{I}$ additive if and only if $\phi$ is a $\rho$ metric measure;

$.2 \quad \mathfrak{I}$ is a regular topology;

$.3 \mathscr{S}$ is normal.

A well known theorem is the following: 
2.7. THEOREM. If $\rho$ metrizes $\mathscr{S}$ and $\phi$ measures $\mathscr{S}$ then $\phi$ is a $\rho$ metric measure if and only if the $\rho$-open sets are $\phi$ measurable.

The primary aim of this section is to generalize Theorem 2.7 to the case where the metric space is replaced by a regular, $\phi$ Normal, topology. This is done in Theorem 2.19.

2.8. Theorem. If $\phi$ measures $\mathscr{S}, C$ is closed and $C \subset A$ then:

.1 if $A$ is compact then $C$ is compact;

.2 if $A$ is $\phi$ compact then $C$ is $\phi$ compact.

Proof. .1 is well known.

Suppose $F \subset \mathfrak{I}, C \subset \sigma F, \psi$ is a submeasure of $\phi, \psi^{\prime}=$ section $\psi C$, $0<\varepsilon<\infty, F^{\prime}=\mathrm{E} B(B \in F$ or $B=\mathscr{S} \sim C)$. Since $A \subset \sigma F^{\prime \prime}, F^{\prime} \subset \mathfrak{I}$, $\psi^{\prime}$ is a submeasure of $\phi$, and $A$ is $\phi$ compact, we can and do choose such a finite subfamily $H^{\prime}$ of $F^{\prime}$ that

$$
\psi^{\prime}(A) \leqq \psi^{\prime}\left(A \sigma H^{\prime}\right)+\varepsilon .
$$

Letting $H=\mathrm{E} B\left(B \in H^{\prime}\right.$ and $\left.B \neq \mathscr{S} \sim C\right)$, it follows that $H$ is a finite subfamily of $F$, and

$$
\begin{aligned}
\psi(C) & =\psi^{\prime}(A) \leqq \psi^{\prime}\left(A \sigma H^{\prime}\right)+\varepsilon \\
& =\psi^{\prime}(A \sigma H)+\varepsilon=\psi(C \sigma H)+\varepsilon .
\end{aligned}
$$

Consequently $C$ is $\phi$ compact, and .2 is proved.

2.9. THEOREM. If $\mathfrak{I}$ is regular, and $\phi$ measures $\mathscr{S}$ then

.1 if $A$ is compact then $A$ is normal,

.2 if $A$ is $\phi$ compact then $A$ is $\phi$ normal.

Proof. .1 is well known.

Suppose $A$ is $\phi$ compact, $C \subset A, C$ is closed, $C \subset B \in \mathfrak{I}, \psi$ is a submeasure of $\phi$, and $\varepsilon>0$. First use the fact that $\mathfrak{I}$ is regular to secure such a function $f$ on $C$ that $f(x) \in \mathfrak{I}, x \in f(x)$, Closure $f(x) \subset B$ for each $x \in C$. Now again use the regularity of $\mathfrak{I}$ to secure such a function $g$ on $C$ that $g(x) \in \mathfrak{I}, x \in g(x)$, Closure $g(x) \subset f(x)$ for each $x \in C$. Now use the (2.8) facts that $C$ is $\phi$ compact and $C \subset \mathrm{U} x \in C g(x)$ to secure such a finite subset $Q$ of $C$ that

$$
\psi(C) \leqq \psi(C \cap \bigcup x \in Q g(x))+\varepsilon .
$$


Let

$$
D=\bigcup x \in Q f(x), \quad C^{\prime}=\bigcup x \in Q(C \cap \text { Closure } g(x))
$$

and observe that $D \in \mathfrak{T}, C^{\prime}$ is closed, $C^{\prime} \subset C$,

$$
C^{\prime}=\mathrm{U} x \in Q(C \cap \text { Closure } g(x)) \subset \mathrm{U} x \in Q f(x)=D,
$$

Closure $D=$ Closure $\bigcup x \in Q f(x)=\bigcup x \in Q$ Closure $f(x) \subset B$, and

$$
\begin{aligned}
\psi(C) & \leqq \psi(C \cap \bigcup x \in Q g(x))+\varepsilon \\
& \leqq \psi(C \cap \bigcup x \in Q \text { Closure } g(x))+\varepsilon \\
& =\psi\left(C^{\prime}\right)+\varepsilon .
\end{aligned}
$$

Consequently $A$ is $\phi$ normal and the proof is complete.

The following theorem is given in Chapter 5 of [3].

2.10. THEOREM. If $\phi$ measures $\mathscr{S}$ and if for each $n \in \omega$ and each submeasure $\psi$ of $\phi$,

$$
A_{n} \subset A_{n+1} \subset \mathscr{S},
$$

and

$$
\psi\left(A_{n} \cup\left(\mathscr{S} \sim A_{n+1}\right)\right)=\psi\left(A_{n}\right)^{\circ}+\psi\left(\mathscr{S} \sim A_{n+1}\right)
$$

then

$.1 \bigcup n \in \omega A_{n}$ is $\phi$ measurable, and

$.2 \theta\left(\bigcup n \in \in A_{n}\right)=\lim _{n \rightarrow \infty} \theta\left(A_{n}\right)$, whenever $\theta$ is a submeasure of $\phi$.

By considering complements one easily established the following corollary of 2.10 .

2.11. THEOREM. If $\phi$ measures $\mathscr{S}$ and if for each $n \in \omega$ and each submeasure $\dot{\psi}$ of $\phi$

$$
A_{n+1} \subset A_{n} \subset \mathscr{S},
$$

and

$$
\psi\left(A_{n+1} \cup\left(\mathscr{S} \sim A_{n}\right)\right)=\psi\left(A_{n+1}\right)+\psi\left(\mathscr{S} \sim A_{n}\right)
$$

then

$.1 \cap n \in \omega A_{n}$ is $\phi$ measurable, and

$.2 \theta\left(\bigcap n \in \omega A_{n}\right)=\lim _{n \rightarrow \infty} \theta\left(A_{n}\right)$, whenever $\theta$ is a submeasure of $\phi$.

The following lemma is easily verified. 
2.12. Lemma. If $\phi$ measures $\mathscr{S}$ then $\phi$ is $\mathfrak{I}$ additive if and only if $\psi$ is $\mathfrak{I}$ additive for each submeasure $\psi$ of $\phi$.

2.13. Theorem. If $\phi$ measures $\mathscr{S}, \phi$ is $\mathfrak{I}$ additive, $A_{n} \subset \mathscr{S}$, and Closure $A_{n+1} \cap$ Closure $\left(\mathscr{S} \sim A_{n}\right)=0$ for each $n \in \omega$, then

$.1 \bigcap n \in \omega A_{n}$ is $\phi$ measurable, and

$.2 \theta\left(\bigcap n \in \omega A_{n}\right)=\lim _{n \rightarrow \infty} \theta\left(A_{n}\right)$, whenever $\theta$ is a submeasure of $\phi$.

Proof. Observe that $A_{n+1} \subset A_{n} \subset \mathscr{S}$, for $n \in \omega$. Let $\psi$ be a submeasure of $\phi$. Since, by 2.12, $\psi$ is $\mathfrak{I}$ additive, it follows that

$$
\psi\left(A_{n+1} \cup\left(\mathscr{S} \sim A_{n}\right)\right)=\psi\left(A_{n+1}\right)+\psi\left(\mathscr{S} \sim A_{n}\right)
$$

for each $n \in \omega$. Application of 2.11 completes the proof.

2.14. THEOREM. If $\phi$ measures $\mathscr{S}, \phi(\mathscr{S})<\infty, \phi$ is $\mathfrak{I}$ additive, $C$ is $\phi$ normal, $C \subset B \in \mathfrak{I}$, and $0<\varepsilon<\infty$, then there exist sets $C^{\prime}$ and $D$ such that $C^{\prime}$ is $\phi$ measurable, $C^{\prime} \in$ Gdelta, $C^{\prime} \subset C, D \in \mathfrak{I}, C^{\prime} \subset D$, Closure $D \subset B$, and $\phi\left(C \sim C^{\prime}\right) \leqq \varepsilon$.

Proof. Since $\phi(\mathscr{S})<\infty$ it follows that $\phi$ is a submeasure of $\phi$. We use the facts that $C$ is $\phi$ normal and $C \subset B$ to inductively obtain such sequences $c^{\prime}$ and $d$ that $c_{0}^{\prime}=C, d_{0}=B, c_{n}^{\prime}$ is closed, $d_{n} \in \mathfrak{I}$, $c_{n+1}^{\prime} \subset c_{n}^{\prime} \subset d_{n}$, Closure $d_{n+1} \subset d_{n}$, and $\phi\left(c_{n}^{\prime}\right) \leqq \phi\left(c_{n+1}^{\prime}\right)+\varepsilon / 2^{n+1}$, for each $n \in \omega$.

Let $C^{\prime}=\bigcap n \in \omega d_{n}, D=d_{1}$. Since

$$
\text { Closure } d_{n+1} \cap \text { Closure }\left(\mathscr{S} \sim d_{n}\right)=0
$$

for each $n \in \omega$, it follows from 2.13.1 that $C^{\prime}$ is $\phi$ measurable. Also

$$
C^{\prime}=\bigcap n \in \omega d_{n}=\bigcap n \in \omega \text { Closure } d_{n+1}
$$

is closed, $C^{\prime} \in$ Gdelta, $D \in \mathfrak{I}, C^{\prime} \subset C, C^{\prime} \subset d_{1}=D$ and Closure $D=$ Closure $d_{1} \subset d_{0}=B$.

We now use induction to deduce that, for any $m \in \omega$,

$$
\begin{aligned}
\phi(C) & =\phi\left(c_{0}^{\prime}\right) \leqq \phi\left(c_{m}^{\prime}\right)+\sum n \in \omega\left(\varepsilon / 2^{n+1}\right) \\
& \leqq \phi\left(c_{m}^{\prime}\right)+\varepsilon .
\end{aligned}
$$

Since $c_{m}^{\prime} \subset d_{m}, c_{m}^{\prime} \subset C$, and $c_{m}^{\prime} \subset C \cap d_{m}$, for each $m \in \omega$, it follows from (1) that

$$
\phi(C) \leqq \phi\left(c_{m}^{\prime}\right)+\varepsilon \leqq \phi\left(C \cap d_{m}\right)+\varepsilon
$$


for each $m \in \omega$. Let $\theta=$ section $\phi C$ and, with the help of 2.13.2. observe that

$$
\begin{aligned}
\phi\left(C C^{\prime}\right) & =\theta\left(C^{\prime}\right) \\
& =\lim _{m \rightarrow \infty} \theta\left(d_{m}\right) \\
& =\lim _{m \rightarrow \infty} \phi\left(C d_{m}\right) \\
\geqq & \lim _{m \rightarrow \infty} \phi(C)-\varepsilon \\
& =\phi(C)-\varepsilon,
\end{aligned}
$$

and, since $C^{\prime}$ is $\phi$ measurable,

$$
\phi\left(C \sim C^{\prime}\right)=\phi(C)-\phi\left(C C^{\prime}\right) \leqq \varepsilon .
$$

2.15. Theorem. If $\phi$ measures $\mathscr{S}, \phi(\mathscr{S})<\infty, \phi$ is $\mathfrak{I}$ additive, $C$ is $\phi$ normal, and $C \subset B \in \mathfrak{I}$, then for some $\phi$ measurable set $K$, $C \subset K \subset B$.

Proof. Repeatedly use 2.14 to secure such a sequence, $k$, of $\phi$ measurable sets that $k_{n} \subset B$, and $\phi\left(C \sim k_{n}\right) \leqq\left(1 / 2^{n}\right)$ for each $n \in \omega$. Let

$$
K^{\prime}=\bigcup n \in \omega k_{n}, \quad K=K^{\prime} \cup C .
$$

Thus $K^{\prime}$ is $\phi$ measurable, $K \subset B$,

$$
0 \leqq \phi\left(C \sim K^{\prime}\right) \leqq \phi\left(C \sim k_{n}\right) \leqq\left(1 / 2^{n}\right)
$$

for each $n \in \omega, \phi\left(C \sim K^{\prime}\right)=0, C \sim K$ is $\phi$ measurable, $K=K^{\prime} \cup\left(C \sim K^{\prime}\right)$ is $\phi$ measurable and $C \subset K \subset B$.

The following lemma is well known.

2.16. Lemma. If $\phi$ measures $\mathscr{S}$ and $A \subset \mathscr{S}$ then $A$ is $\phi$ measurable if and only if $A$ is $\psi$ measurable for each submeasure $\psi$ of $\phi$.

2.17. THEOREM. If $\phi$ measures $\mathscr{S}, \phi$ is $\mathfrak{I}$ additive and $\mathscr{S}$ is $\phi$ normal then Fsigma $\subset$ measurable $\phi$.

Proof. Let $B \in$ Fsigma, and let $\psi$ be a submeasure of $\phi$. Choose such a countable subfamily $F$ of closed sets that $B=\sigma F$. Check that $\psi(\mathscr{S})<\infty, \psi$ is $\mathbb{I}$ additive, $C$ is $\phi$ normal, and $C$ is $\psi$ normal, for each $C \in F$.

Thus we can and do use 2.15 to secure such a function $K$ on $F$ that $K(C)$ is $\psi$ measurable and $C \subset K(C) \subset B$ for each $C \in F$. It follows that 


$$
\begin{aligned}
& B=\sigma F=\bigcup C \in F C \\
& \subset \cup C \in F K(C) \\
& \subset B \text {, } \\
& B=\bigcup C \in F K(C) \text {, }
\end{aligned}
$$

$B$ is $\psi$ measurable.

Thus $B$ is $\psi$ measurable for each submeasure $\dot{\psi}$ of $\phi$, and, by 2.16, $B$ is $\phi$ measurable.

The desired conclusion is at hand.

2.18. Theorem. If $\phi$ measures $\mathscr{S}, \phi(\mathscr{S})<\infty, C$ is $\phi$ Normal $C \subset B \in \mathfrak{I}$, and $0<\varepsilon<\infty$, then there exists such a member $C^{\prime}$ of Gdelta that $C^{\prime} \subset B$ and $\phi\left(C \sim C^{\prime}\right) \leqq \varepsilon$.

Proof. Repeatedly use the fact that $C$ is $\phi$ Normal to obtain such sequences $c^{\prime}$ and $d$ that $c_{0}^{\prime}=C, d_{0}=B, c_{n}^{\prime}$ is closed, $d_{n} \in \mathfrak{I}$, $c_{n+1}^{\prime} \subset c_{n}^{\prime} \subset d_{n}$, Closure $d_{n+1} \subset d_{n}$, and

$$
\phi\left(c_{n}^{\prime} \sim c_{n+1}^{\prime}\right) \leqq\left(\varepsilon / 2^{n+1}\right),
$$

for each $n \in \omega$.

Let $C^{\prime}=\bigcap n \in \omega d_{n}$ and observe that $C^{\prime} \in$ Gdelta, $C^{\prime} \subset B$,

$$
\begin{aligned}
\phi\left(C \sim C^{\prime}\right) & =\phi\left(C \sim \cap n \in \omega d_{n}\right) \\
& =\phi\left(\bigcup n \in \omega\left(C \sim d_{n}\right)\right) \\
& \leqq \phi\left(\bigcup n \in \omega\left(C \sim c_{n}^{\prime}\right)\right) \\
& =\phi\left(\bigcup n \in \omega\left(c_{0}^{\prime} \sim c_{n}^{\prime}\right)\right) \\
& =\phi\left(\bigcup n \in \omega\left(c_{n}^{\prime} \sim c_{n+1}^{\prime}\right)\right) \\
& \left.\leqq \sum n \in \omega \phi\left(c_{n}^{\prime} \sim c_{n+1}^{\prime}\right)\right) \\
& \leqq \sum n \in \omega\left(\varepsilon / 2^{n+1}\right)=\varepsilon .
\end{aligned}
$$

Thus $\phi\left(C \sim C^{\prime}\right) \leqq \varepsilon$, and the proof is complete.

2.19. Theorem. If $\phi$ measures $\mathscr{S}$, and $\mathscr{S}$ is $\phi$ Normal then Fsigma $\subset$ measurable $\phi$

if and only if $\phi$ is $\mathfrak{I}$ additive.

Proof. If $\phi$ is $\mathfrak{I}$ additive, it follows from 2.17 that

Fsigma $\subset$ measurable $\phi$,

since (2.5.3) a set which is $\phi$ Normal is also $\phi$ normal.

Now suppose that Fsigma $\subset$ measurable $\phi$. Let $\dot{\psi}$ be a sub- 
measure of $\phi, 0<\varepsilon<\infty, A \subset \mathscr{S}, B \subset \mathscr{S}, \bar{A}=$ Closure $A, \bar{B}=$ Closure $B, \alpha=\mathscr{S} \sim \bar{B}$, and suppose that $\bar{A} \bar{B}=0$.

Since $\bar{A} \subset \alpha$ we may use 2.18 to secure such a member $C^{\prime}$ of Gdelta that $C^{\prime} \subset \alpha$ and $\psi\left(\bar{A} \sim C^{\prime}\right) \leqq \varepsilon$. Thus $\psi\left(A \sim C^{\prime}\right) \leqq \varepsilon,(A \cup B) C^{\prime}=$ $A C^{\prime},(A \cup B) \sim C^{\prime}=\left(A \sim C^{\prime}\right) \cup B$, and, since $C^{\prime} \in$ Fsigma $\subset$ measurable $\phi$,

$$
\begin{aligned}
\psi(A \cup B) & =\psi\left((A \cup B) C^{\prime}+\psi\left((A \cup B) \sim C^{\prime}\right)\right. \\
& =\psi\left(A C^{\prime}\right)+\psi\left(\left(A \sim C^{\prime}\right) \cup B\right) \\
& \geqq \psi\left(A C^{\prime}\right)+\psi(B) \\
& =\psi(A)-\psi\left(A \sim C^{\prime}\right)+\psi(B) \\
& \geqq \psi(A)+\psi(B)-\varepsilon .
\end{aligned}
$$

Thus $\psi(A \cup B) \geqq \psi(A)+\psi(B)$, and since $\psi(A \cup B) \leqq \psi(A)+\psi(B)$, it follows that

$$
\psi(A \cup B)=\psi(A)+\psi(B) .
$$

Therefore $\phi$ is $\mathfrak{T}$ additive, and the proof is complete.

2.20. REMARK. Since any metric space is normal and therefore (2.52) $\phi$ Normal and since every open set of a metric space is an $F_{\sigma}$, it follows that 2.19 is a generalization of the following well known theorem.

THEOREM. If $\rho$ metrizes $\mathscr{S}, \phi$ measures $\mathscr{S}$, and $\mathfrak{I}$ is the family of $\rho$-open sets, then $\mathfrak{I} \subset$ measurable $\phi$ if and only if $\phi(A \cup B)=$ $\phi(A)+\phi(A)$ whenever $A$ and $B$ are a positive $\rho$-distance apart.

We shall use 2.17 in the next section where the topological space is not known to be normal but is $\phi$ normal for the measure $\phi$ that is constructed there.

\section{Measure construction.}

It is well known that the set function, $\theta=\operatorname{msm} g \rho H$, defined in 3.2 below, is a Borel measure (i.e., the $\rho$-open sets are $\theta$ measurable) in case $\rho$ metrizes $\mathscr{S}$ and $g$ is a nonnegative function of $H$. It is the purpose of this section to generalize this result to the topolagical case by defining a measure, $\phi=$ mst $g \mathfrak{T} H$, for which the open $F_{\sigma}$ 's are $\phi$-measurable whenever $\mathfrak{I}$ is regular, and which is equal to $\theta$ in case $\mathfrak{T}$ is metrizable.

\subsection{Definitions.}

.1 mss $g \mathscr{S} H=$ the function $\psi$, on the subsets of $\mathscr{S}$, such 
that if $A \subset \mathscr{S}$ then $\psi(A)$ is the infimum of numbers of the form

$$
\sum B \in F g(B)
$$

where $F$ is such a countable subfamily of $H$ that $A \subset \sigma F$.

In connection with 3.1. we would like to remind the reader that an empty infimum is $\infty$.

$.2 \mathrm{msm} g \rho H=$ the function $\theta$, on the subsets of $\mathscr{S}$, such that, if $A \subset \mathscr{S}$ then

$$
\theta(A)=\lim _{n \rightarrow \infty} \operatorname{mss} g \mathscr{S} H_{n}(A),
$$

where $H_{n}$ consists of those members of $H$ whose $\rho$-diameter is less than $1 / 2^{n}$, for each $n$.

$.3 \operatorname{msf} g F H=\operatorname{mss} g \mathscr{S} H_{F}$, where

$$
H_{F}=H \cap \mathrm{E} D(D \subset B \text { for some } B \in F) \text {. }
$$

.4 Cover $\mathfrak{I}=\mathrm{E} F(F \subset \mathfrak{I}$ and $\sigma F=\sigma \mathfrak{I})$.

Thus, $E \in$ Cover $\mathfrak{I}$ if and only if $F \subset \mathfrak{I}$ and $F$ covers the space covered by $\mathfrak{T}$.

.5 mst $g \mathfrak{T} H=$ the function $\phi$ on the subsets of $\mathscr{S}$ such that, if $A \subset \mathscr{S}$ then

$$
\phi(A)=\sup F \in \operatorname{Cover} \mathfrak{T} \phi_{F}(A)
$$

where $\phi_{F}=\operatorname{msf} g F H$, for each $F \in$ Cover $\mathfrak{T}$.

$.6 F$ is a refinement of $F^{\prime}$ if and only if each member of $F$ is a subset of some member of $F^{\prime \prime}$.

$.7 F \cap \cap F^{\prime}=\mathrm{E} B\left(B=\alpha \beta\right.$ for some $\alpha \in F$ and some $\left.\beta \in F^{\prime}\right)$.

3.1. Lemma. If $F \in$ Cover $\mathfrak{I}, F^{\prime} \in$ Cover $\mathfrak{T}$, and $F^{\prime \prime}=F \cap \cap F^{\prime}$, then $F^{\prime \prime} \in$ Cover $\mathfrak{I}$ and $F^{\prime \prime}$ is a refinement of $F$.

The following theorem is well known.

3.2. THEOREM. If $g$ is a nonnegative function on $H$, and $\psi=$ mss $g \mathscr{S} H$ then:

$.1 \psi$ measures $\mathscr{S}$;

.2 if $H \subset H^{\prime}$ and $\psi^{\prime}=\operatorname{mss} g \mathscr{S} H^{\prime}$, then $\psi^{\prime}(A) \leqq \psi(A)$ for 
$A \subset \mathscr{S}$

.3 if $\psi(A)<\infty$ and $0<\varepsilon<\infty$ then there exists such a countable subfamily $G$ of $H$ that $A \subset \sigma G$ and $\sum D \in G g(D) \leqq \psi(A)+\varepsilon$.

3.3. THEOREM. If $g$ is a nonnegative function of $H, F$ is a refinement of $F^{\prime}, \phi_{F}=\operatorname{msf} g F H$, and $\phi_{F^{\prime}}=\operatorname{msf} g F^{\prime} H$, then

$$
\phi_{F^{\prime}}(A) \leqq \phi_{F}(A)
$$

for each $A \subset S$.

Proof. Let

$$
\begin{aligned}
H_{F} & =H \cap \mathrm{E} B(B \subset D \text { for some } D \in F), \\
H_{F^{\prime}} & =H \cap \mathrm{E} B\left(B \subset D \text { for some } D \in F^{\prime}\right),
\end{aligned}
$$

and note that $\phi_{F}=\operatorname{mss} g \mathscr{S} H_{F}$. Application of 3.2.2 completes the proof.

The following theorem is well known.

3.4. THEOREM. If $F$ is nonempty, $\psi$ measures $\mathscr{S}$ for each $\psi \in F$, and

$$
\phi(D)=\sup \psi \in F \psi(D)
$$

whenever $D \subset \mathscr{S}$, then:

$.1 \phi$ measures $\mathscr{S}$;

.2 if for each $\psi^{\prime} \in F$ and each $\psi^{\prime \prime} \in F$, there exists $a \psi \in F$ for which

$$
\psi^{\prime}(D) \leqq \psi(D) \text { and } \psi^{\prime \prime}(D) \leqq \psi(D)
$$

whenever $D \subset \mathscr{S}$, then

$$
\bigcap \psi \in F \text { measurable } \psi \subset \text { measurable } \phi \text {. }
$$

3.5. THEOREM. If $\mathfrak{T}$ is a regular topology, $g$ is a nonnegative: function on $H$, and $\phi=$ mst $g \mathfrak{T} H$ then Fsigma $\subset$ measurable $\phi$.

Proof. Let $\phi_{F}=\operatorname{msf} g F H$ for each $F \in$ Cover $\mathfrak{T}$. Thus

$$
\phi(A)=\sup F \in \operatorname{Cover} \mathfrak{I} \phi_{F}(A)
$$

whenever $A \subset \mathscr{S}$. The proof is completed in six parts.

PART I. $\phi$ measures $\mathscr{S}$. 
Proof. Since, by 3.2.1, $\phi_{F}$ measures $\mathscr{S}$, for each $F \in$ Cover $\mathfrak{I}$, and since Cover $\mathfrak{I}$ is not empty, it follows from 3.4.1 that $\phi$ measures $\mathscr{S}$.

PART II. If $F \in$ Cover $\mathfrak{I}, \phi(A)<\infty$, and $0<\varepsilon<\infty$ then there exists such a refinement $F^{\prime}$ of $F$ that $F^{\prime} \in \operatorname{Cover} \mathfrak{I}$, and $\phi(A) \leqq$ $\phi_{F^{\prime}}(A)+\varepsilon$.

Proof. Choose such a member $F^{\prime \prime}$ of Cover $\mathfrak{I}$ that $\phi(A) \leqq$ $\phi_{F^{\prime \prime}}(A)+\varepsilon$, and let $F^{\prime}=F \cap \cap F^{\prime \prime}$ (see Definition 3.0.7). Thus $S \subset \sigma F^{\prime}$, $F^{\prime} \subset \mathfrak{I}, F^{\prime} \in$ Cover $\mathfrak{I}$, and using 3.1 and 3.3 , we infer that $F^{\prime}$ is a refinement of $F, F^{\prime \prime}$ is a refinement of $F^{\prime \prime}$, and

$$
\phi(A) \leqq \phi_{F^{\prime \prime}}(A)+\varepsilon \leqq \phi_{F^{\prime}}(A)+\varepsilon .
$$

PART III. $\mathscr{S}$ is $\phi$ compact.

Proof. Recall definition 2.4.2. Let $F \in$ Cover $\mathfrak{I}, \phi(T)<\infty, \psi=$ section $\phi T$, and $0<\varepsilon<\infty$. Use Part II to choose such a refinement $F^{\prime}$ of $F$ that $F^{\prime} \in$ Cover $\mathfrak{I}$ and

$$
\phi(T) \leqq \phi_{F^{\prime}}(T)+\varepsilon / 2<\infty .
$$

Now use 3.2.3 and definition 3.0.3 to secure such a countable subfamily $H^{\prime}$ of $H$ that $T \subset \sigma H^{\prime}, H^{\prime}$ is a refinement of $F^{\prime}$, and $\sum D \in H^{\prime} g(D)<\infty$; and let $H^{\prime \prime}$ be such a finite subfamily of $H^{\prime}$ that

$$
\sum D \in\left(H^{\prime} \sim H^{\prime \prime}\right) g(D) \leqq \varepsilon / 2 .
$$

Since $H^{\prime \prime}$ is a refinement of $F$, choose such a finite subfamily $G$ of $F$ that $H^{\prime \prime}$ is a refinement of $G$. Thus

$$
\begin{aligned}
\phi_{F^{\prime}}(T) & \leqq \phi_{F^{\prime}}\left(T \sigma H^{\prime \prime}\right)+\phi_{F^{\prime}}\left(T \sigma\left(H^{\prime} \sim H^{\prime \prime}\right)\right) \\
& \leqq \phi\left(T \sigma H^{\prime \prime}\right)+\phi_{F^{\prime}}\left(\sigma\left(H^{\prime} \sim H^{\prime \prime}\right)\right) \\
& \leqq \phi(T \sigma G)+\sum D \in\left(H^{\prime} \sim H^{\prime \prime}\right) g(D) \\
& \leqq \psi(T)+\varepsilon / 2, \\
\psi(\mathscr{S}) & =\phi(T) \leqq \phi_{F^{\prime}}(T)+\varepsilon / 2 \\
& \leqq \psi(T)+\varepsilon .
\end{aligned}
$$

Thus $\mathscr{S}$ is $\phi$ compact.

PART IV. $\phi$ is $\mathfrak{I}$ additive.

Proof. Recall definition 2.4.6. Let $\bar{A}=$ Closure $A, \bar{B}=$ Closure $B$, $\bar{A} \bar{B}=0$. If $\phi(A \cup B)=\infty$ then $\phi(A \cup B)=\phi(A)+\phi(B)$. 
Now assume $\phi(A \cup B)<\infty, 0<\varepsilon<\infty$, and select such members $G^{\prime}$ and $G^{\prime \prime}$ of Cover $\mathfrak{I}$ that

$$
\phi(A) \leqq \phi_{\theta^{\prime}}(A)+\varepsilon / 3, \quad \phi(B) \leqq \phi_{a^{\prime \prime}}(B)+\varepsilon / 3,
$$

and let

$$
\begin{aligned}
F^{\prime} & =\mathrm{E} \alpha\left(\alpha=B \cap(\mathscr{S} \sim \bar{B}) \text { for some } \beta \in G^{\prime}\right), \\
F^{\prime \prime} & =\mathrm{E} \alpha\left(\alpha=\beta \cap(\mathscr{S} \sim \bar{A}) \text { for some } \beta \in G^{\prime \prime}\right), \\
F & =F^{\prime} \cup F^{\prime \prime} .
\end{aligned}
$$

Thus:

$$
\begin{gathered}
F \subset \mathfrak{T} ; \mathscr{S} \subset \sigma F ; \quad F \in \text { Cover } \mathfrak{T} ; \\
\text { if } D \in F \text { and } D A \neq 0 \text { then } D \in F^{\prime} ; \\
\text { if } D \in F \text { and } D B \neq 0 \text { then } D \in F^{\prime \prime} .
\end{gathered}
$$

Now use 3.2.3 to secure such a countable subfamily $H^{\prime \prime \prime}$ of $H$ that $H^{\prime \prime \prime}$ is a refinement of $F, A \cup B \subset H^{\prime \prime \prime}$, and

$$
\sum D \in H^{\prime \prime \prime} g(D) \leqq \phi_{F}(A \cup B)+\varepsilon / 3 ;
$$

infer from (2) that

$$
\begin{aligned}
& \text { if } D \in H^{\prime \prime \prime} \text { and } D A \neq 0 \text { then } D B=0 \\
& \text { if } D \in H^{\prime \prime \prime} \text { and } D B \neq 0 \text { then } D A=0 \text {; }
\end{aligned}
$$

let $H^{\prime}=H^{\prime \prime \prime} \cap \mathrm{E} D(D A \neq 0), H^{\prime \prime}=H^{\prime \prime \prime} \cap \mathrm{E} D(D B \neq 0)$.

Thus $H^{\prime} \cup H^{\prime \prime} \subset H^{\prime \prime \prime} \subset H, H^{\prime} \cup H^{\prime \prime}$ is countable, $A \subset \sigma H^{\prime}, B \subset \sigma H^{\prime \prime}$, $H^{\prime} H^{\prime \prime}=0, H^{\prime}$ is a refinement of $G^{\prime}, H^{\prime \prime}$ is a refinement of $G^{\prime \prime}$, and

$$
\phi_{G^{\prime}}(A) \leqq \sum D \in H^{\prime} g(D), \quad \phi_{\sigma^{\prime \prime}}(B) \leqq \sum D \in H^{\prime \prime} g(D) \text {. }
$$

Consequently, with the help of (1), (4) and (3), we deduce that

$$
\begin{aligned}
\phi(A)+\phi(\mathrm{B}) & \leqq \phi_{\theta^{\prime}}(A)+\phi_{\theta^{\prime \prime}}(B)+2 \varepsilon / 3 \\
& \leqq \sum D \in H^{\prime} g(D)+\sum D \in H^{\prime \prime} g(D)+2 \varepsilon / 3 \\
& \leqq \sum D \in H^{\prime \prime \prime} g(D)+2 \varepsilon / 3 \\
& \leqq \phi_{F}(A \cup B)+\varepsilon / 3+2 \varepsilon / 3 \\
& \leqq \phi(A \cup B)+\varepsilon .
\end{aligned}
$$

Thus, if $\phi(A \cup B)<\infty$,

$$
\phi(A)+\phi(B) \leqq \phi(A \cup B) .
$$

Therefore

$$
\phi(A \cup B)=\phi(A)+\phi(B),
$$

whenever $A \subset \mathscr{S}, B \subset \mathscr{S}$, and $\phi$ is $\mathfrak{T}$ additive. 
PART V. $\mathscr{S}$ is $\phi$ normal.

Proof. Recall definition 2.4.4, use part IV and 2.9.2.

PART VI. Fsigma $\subset$ measurable $\phi$.

Proof. By parts IV and V, $\phi$ is $\mathfrak{I}$ additive and $\mathscr{S}$ is $\phi$ normal. Application of 2.17 completes the proof.

The reader will observe that the regularity of $\mathfrak{I}$ was not used in the proofs of Parts I-III.

3.6. Lemma. If $\rho$ metrizes $\mathscr{S}, \mathfrak{T}$ is the family of $\rho$-open sets, $F \subset \mathfrak{I}, n \in \omega$, and $G_{n}=\mathrm{E} D$ (the $\rho$-diameter of $\left.D<1 / 2^{n}\right)$, then:

\section{$.1 \mathfrak{I} \cap G_{n} \in$ Cover $\mathfrak{I}$;}

.2 if $H^{\prime} \subset G_{n}$, if $D A \neq 0$ whenever $D \in H^{\prime}$, and if for each $x \in A$ there exists a $B \in F$ such that

$$
\text { (the } \rho \text {-distance from } x \text { to } \mathscr{S} \sim B \text { ) }>1 / 2^{n},
$$

then $H^{\prime}$ is a refinement of $F$;

$.3 D \in G_{n}$ if, and only if, $D \subset B \in G_{n}$ for some $B \in \mathfrak{T}$.

Proof. Suppose $D \in G_{n}$, and let $d=$ the $\rho$-diameter of $D, \delta=$ $1 / 2^{n}-d$, and

$$
B=\operatorname{E} x(\rho(x, y)<\delta / 3 \text { for some } y \in D) .
$$

Thus, we have immediately that $B \in \mathfrak{I}, D \subset B$, and one can show that $B \in G_{n}$.

Therefore if $D \in G_{n}$ then $D \subset B \in G_{n}$ for some $B \in \mathfrak{T}$.

The remainder of the proof is straightforward.

3.7. THEOREM. If $\rho$ metrizes $\mathscr{S}$, $\mathfrak{I}$ is the family of all $\rho$-open sets, $F \subset \mathfrak{I}, g$ is a nonnegative function on $H, G_{n}=\mathrm{E} D(($ the $\rho$-diameter of $\left.D)<1 / 2^{n}\right)$ for each $n \in \omega$, and $\theta=\operatorname{msm} g \rho H$, then:

$.0 \mathfrak{I} \subset$ measurable $\phi$;

.1 if $\theta(A)<\infty, n \in \omega$, and $0<\varepsilon<\infty$, then there exists a countable subfamily $H^{\prime}$ of $H$ for which $H^{\prime} \subset G_{n}, A \subset H^{\prime}$, and $\sum D \in H^{\prime} g(D) \leqq \theta(A)+\varepsilon ;$ 
.2 if $\theta(A)<\infty, n \in \omega, 0<\varepsilon<\infty$, and if for each $x \in A$ there exists a $B \in F$ such that

(the $\rho$-distance from $x$ to $\mathscr{S} \sim B) \geqq 1 / 2^{n}$,

then there exists a countable subfamily $H^{\prime}$ of $H$ for which $H^{\prime} \subset G_{n}$, $A \subset \sigma H^{\prime}, H^{\prime}$ is a refinement of $F$, and $\Sigma D \in H^{\prime} g(D) \leqq \theta(A)+\varepsilon$.

Proof. .0 is well known.

Proof .1 Let $\theta_{n}=\operatorname{mss} g \mathscr{S}\left(H G_{n}\right)$. Thus $\theta_{n}(A) \leqq \theta(A)<\infty$, and we can use 3.2.3 to secure such a countable subfamily $H^{\prime}$ of $\left(H \cap G_{n}\right)$ that $A \subset \sigma H^{\prime}$ and $\sum D \in H^{\prime} g(D) \leqq \theta_{n}(A)+\varepsilon$. Since $\theta_{n}(A) \leqq \theta(A)$ the proof is complete.

Proof .2 Use .1 to obtain such a countable subfamily $H^{\prime \prime}$ of $\left(H G_{n}\right)$ that $A \subset \sigma H^{\prime \prime}$ and $\sum D \in H^{\prime \prime} g(D) \leqq \theta(A)+\varepsilon$, and let $H^{\prime}=$ $H^{\prime \prime} \cap \mathrm{E} D(D A \neq 0)$.

Thus $A \subset \sigma H^{\prime}$, and $\sum D \in H^{\prime} g(D) \leqq \sum D \in H^{\prime \prime} g(D) \leqq \theta(A)+\varepsilon$. Also $D A \neq 0$ for each $D \in H^{\prime}$, so by 3.6.2 $H^{\prime}$ is a refinement of $F$.

3.8. THeOREM. If $\rho$ metrizes $\mathscr{S}, \mathfrak{I}$ is the family of all $\rho$-open sets, $g$ is a nonnegative function on $H, \phi=\operatorname{mst} g \mathfrak{I} H$, and $\theta=\operatorname{msm} g \rho H$ then $\phi=\theta$.

Proof. Let $\phi_{F}=\operatorname{msf} g F H$ for each $F \in$ Cover $\mathfrak{I}$, and complete the proof in Parts I and II by showing that $\phi(A) \geqq \theta(A)$, and $\phi(A) \leqq$ $\theta(A)$ whenever $A \subset \mathscr{S}$.

PART I. If $A \subset \mathscr{S}$ then $\phi(A) \geqq \theta(A)$.

Proof. Let

$$
G_{n}=\mathrm{E} D\left((\text { the } \rho \text {-diameter of } D)<1 / 2^{n}\right),
$$

$F_{n}=\mathfrak{T} \cap G_{n}, H_{n}=H \cap G_{n}$, and $\theta_{n}=\operatorname{mss} g \mathscr{S} H_{n}$, for each $n \in \omega$. Thus, for each $n \in \omega, F_{n} \in$ Cover $\mathfrak{T}$, and by definition 3.0.3, and 3.6.3,.

$$
\begin{aligned}
\phi_{F_{n}} & =\operatorname{msf} g F_{n} H \\
& =\operatorname{mss} g \mathscr{S}\left(H \cap \mathrm{E} D\left(D \subset B \text { for some } B \in F_{n}\right)\right) \\
& =\operatorname{mss} \mathrm{g} \mathscr{S} H_{n} \\
& =\theta_{n} .
\end{aligned}
$$

Consequently, 


$$
\begin{aligned}
\phi(A) & =\sup F \in \text { Cover } \mathfrak{T} \phi_{F}(A) \\
& \geqq \sup n \in \omega \phi_{F_{n}}(A) \\
& =\sup n \in \omega \theta_{n}(A) \\
& =\theta(A) .
\end{aligned}
$$

PART II. If $A \subset \mathscr{S}$ then $\phi(A) \leqq \theta(A)$.

Proof. The result being obvious if $\theta(A)=\infty$, we assume that $\theta(A)<\infty$. Let $0<\varepsilon<\infty, F \in$ Cover $\mathfrak{I}$, and let $f^{\prime}$ and $f$ be such sequences that

$$
\begin{aligned}
& f_{n}^{\prime}=\mathrm{U} B \in F \mathrm{E} x\left((\text { the } \rho \text {-distance from } x \text { to } \mathscr{S} \sim B)>1 / 2^{n}\right), \\
& f_{0}=f_{0}^{\prime} \text {, } \\
& f_{n+1}=f_{n+1}^{\prime} \sim f_{n}^{\prime} \text {, }
\end{aligned}
$$

whenever $n \in \omega$. We infer: $f_{n}$ is a $\rho$-Borel set and, by 3.7.0, $f_{n}$ is $\phi$ measurable for each $n \in \omega ; A=\bigcup n \in \omega\left(A f_{n}\right) ; \theta(A)=\sum n \in \omega \theta\left(A f_{n}\right)$; for each $n \in \omega$, for each $x \in\left(A f_{n}\right)$, there is a $B \in F$ for which the distance from $x$ to $\mathscr{S} \sim B$ is greater than $1 / 2^{n}$. Consequently, we can use 3.7.2 to secure such a sequence, $h$, that $h_{n}$ is a countable subfamily of $H, A f_{n} \subset \sigma h_{n}, h_{n}$ is a refinement of $F$, and

$$
\sum D \in h_{n} g(D) \leqq \theta\left(A f_{n}\right)+\varepsilon / 2^{n+1},
$$

whenever $n \in \omega$.

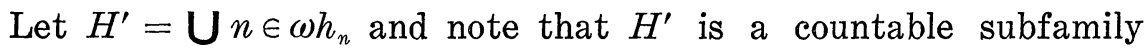
of $H, A=\bigcup n \in \omega\left(A f_{n}\right) \subset \bigcup n \in \omega \sigma h_{n}=\sigma H^{\prime}, H^{\prime}$ is a refinement of $F$, and

$$
\begin{aligned}
\phi_{F}(A) & \leqq \sum D \in H^{\prime} g(D) \leqq \sum n \in \omega \sum D \in h_{n} g(D) \\
& \leqq \sum n \in \omega\left(\theta\left(A f_{n}\right)+\varepsilon / 2^{n+1}\right)=\sum n \in \omega \theta\left(A f_{n}\right)+\varepsilon=\theta(A)+\varepsilon .
\end{aligned}
$$

Thus $\phi_{F}(A) \leqq \theta(A)$ for each $F \in$ Cover $\mathfrak{I}$, and

$$
\begin{aligned}
\phi(A) & =\sup F \in \text { Cover } \mathfrak{I} \phi_{F}(A) \\
& \leqq \theta(A) .
\end{aligned}
$$

3.9. REMARK. Thus, because of Theorems 3.5.3 and 3.8, the topological measure $\phi=$ mst $g \mathfrak{I} H$ is a generalization of the metric measure $\theta=\operatorname{msm} g \rho H$.

\section{REFERENCES}


2. A. P. Morse, Lectures on Real Variables, University of California, Berkeley, 195253.

3. M. E. Munroe, Introduction to Measure and Integration, Addison Wesley, Reading, Mass.

Panoramic Research, Inc., Palo Alto, California

UNIVERSITY OF CALIFORNIA, BERKELEY 


\section{PACIFIC JOURNAL OF MATHEMATICS}

\section{EDITORS}

RalPh S. Phillips

Stanford University

Stanford, California

M. G. Arsove

University of Washington

Seattle 5 , Washington
J. Dugundu

University of Southern California

Los Angeles 7, California

Lowell J. Paige

University of California

Los Angeles 24, California

\section{ASSOCIATE EDITORS}
E. F. BECKENBACH
D. DERRY
H. L. ROYDEN
E. G. STRAUS
T. M. CHERRY
M. OHTSUKA
E. SPANIER
F. WOLF

\section{SUPPORTING INSTITUTIONS}

\author{
UNIVERSITY OF BRITISH COLUMBIA \\ CALIFORNIA INSTITUTE OF TECHNOLOGY \\ UNIVERSITY OF CALIFORNIA \\ MONTANA STATE UNIVERSITY \\ UNIVERSITY OF NEVADA \\ NEW MEXICO STATE UNIVERSITY \\ OREGON STATE UNIVERSITY \\ UNIVERSITY OF OREGON \\ OSAKA UNIVERSITY \\ UNIVERSITY OF SOUTHERN CALIFORNIA
}

\author{
STANFORD UNIVERSITY \\ UNIVERSITY OF TOKYO \\ UNIVERSITY OF UTAH \\ WASHINGTON STATE UNIVERSITY \\ UNIVERSITY OF WASHINGTON \\ AMERICAN MATHEMATICAL SOCIETY \\ CALIFORNIA RESEARCH CORPORATION \\ SPACE TECHNOLOGY LABORATORIES \\ NAVAL ORDNANCE TEST STATION
}

Mathematical papers intended for publication in the Pacific Journal of Mathematrcs should be typewritten (double spaced), and the author should keep a complete copy. Manuscripts may be sent to any one of the four editors. All other communications to the editors should be addressed to the managing editor, L. J. Paige at the University of California, Los Angeles 24, California.

50 reprints per author of each article are furnished free of charge; additional copses may be obtained at cost in multiples of 50 .

The Pacific Journal of Mathematics is published quarterly, in March, June, September, and December. Effective with Volume 13 the price per volume (4 numbers) is $\$ 18.00$; single issues, $\$ 5.00$. Special price for current issues to individual faculty members of supporting institutions and to individual members of the American Mathematical Society: $\$ 8.00$ per volume; single issues $\$ 2.50$. Back numbers are available.

Subscriptions, orders for back numbers, and changes of address should be sent to Pacific Journal of Mathematics, 103 Highland Boulevard, Berkeley 8, California.

Printed at Kokusai Bunken Insatsusha (International Academic Printing Co., Ltd.), No. 6 , 2-chome, Fujimi-cho, Chiyoda-ku, Tokyo, Japan.

\section{PUBLISHED BY PACIFIC JOURNAL OF MATHEMATICS, A NON-PROFIT CORPORATION}

The Supporting Institutions listed above contribute to the cost of publication of this Journal, but they are not owners or publishers and have no responsibility for its content or policies. 


\section{Pacific Journal of Mathematics}

\section{Vol. 13, No. $4 \quad$ June, 1963}

Dallas O. Banks, Bounds for eigenvalues and generalized convexity ........... 1031

Jerrold William Bebernes, A subfunction approach to a boundary value problem for

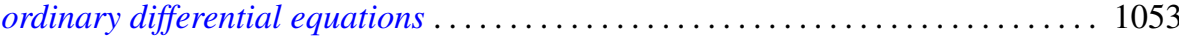

Woodrow Wilson Bledsoe and A. P. Morse, A topological measure construction . . . 1067

George Clements, Entropies of several sets of real valued functions . . . . . . . . . 1085

Sandra Barkdull Cleveland, Homomorphisms of non-commutative *-algebras . . . . . 1097

William John Andrew Culmer and William Ashton Harris, Convergent solutions of

ordinary linear homogeneous difference equations . . . . . . . . . . . . . . . 1111

Ralph DeMarr, Common fixed points for commuting contraction mappings . . . . . . 1139

James Robert Dorroh, Integral equations in normed abelian groups . . . . . . . . 1143

Adriano Mario Garsia, Entropy and singularity of infinite convolutions . . . . . . . 1159

J. J. Gergen, Francis G. Dressel and Wilbur Hallan Purcell, Jr., Convergence of extended Bernstein polynomials in the complex plane ................. 1171

Irving Leonard Glicksberg, A remark on analyticity of function algebras . . . . . . 1181

Charles John August Halberg, Jr., Semigroups of matrices defining linked operators

with different spectra ................................. 1187

Philip Hartman and Nelson Onuchic, On the asymptotic integration of ordinary

differential equations . . . . . . . . . . . . . . . . . . . . . . . . . . . . 1193

Isidore Heller, On a class of equivalent systems of linear inequalities . . . . . . . . . 1209

Joseph Hersch, The method of interior parallels applied to polygonal or multiply

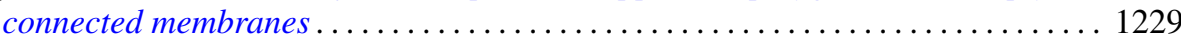

Hans F. Weinberger, An effectless cutting of a vibrating membrane . . . . . . . . . . 1239

Melvin F. Janowitz, Quantifiers and orthomodular lattices ....

Samuel Karlin and Albert Boris J. Novikoff, Generalized convex inequalities . .

Tilla Weinstein, Another conformal structure on immersed surfaces of negative

curvature.

Gregers Louis Krabbe, Spectral permanence of scalar operators

Shige Toshi Kuroda, Finite-dimensional perturbation and a representaion of

scattering operator.

Marvin David Marcus and Afton Herbert Cayford, Equality in certain

inequalities

Joseph Martin, A note on uncountably many disks .

Eugene Kay McLachlan, Extremal elements of the convex cone of semi-norms . . . . 1335

John W. Moon, An extension of Landau's theorem on tournaments . .

Louis Joel Mordell, On the integer solutions of $y(y+1)=x(x$

Kenneth Roy Mount, Some remarks on Fitting's invariants .....

Miroslav Novotný, Über Abbildungen von Mengen ............

Robert Dean Ryan, Conjugate functions in Orlicz spaces.

John Vincent Ryff, On the representation of doubly stochastic operators . . . . . . . . 1379

Donald Ray Sherbert, Banach algebras of Lipschitz functions .

James McLean Sloss, Reflection of biharmonic functions across analytic boundary

conditions with examples.

L. Bruce Treybig, Concerning homogeneity in totally ordered, connected topological space....

John Wermer, The space of real parts of a function algebra...

James Juei-Chin Yeh, Orthogonal developments of functionals and related theorems

in the Wiener space of functions of two variables......... 